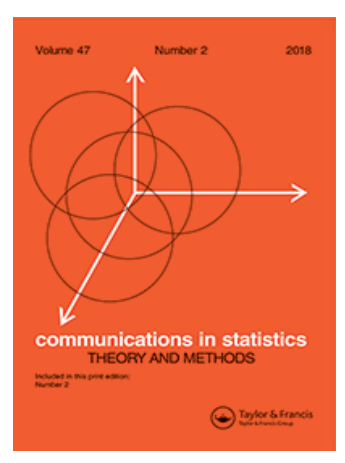

Communications in Statistics - Theory and Methods

ISSN: 0361-0926 (Print) 1532-415X (Online) Journal homepage: http://www.tandfonline.com/loi/lsta20

\title{
Asymptotic estimate of variance with applications to stochastic differential equations arises in mathematical neuroscience
}

\section{Rahman}

To cite this article: M. Rahman (2018) Asymptotic estimate of variance with applications to stochastic differential equations arises in mathematical neuroscience, Communications in Statistics - Theory and Methods, 47:2, 289-306, DOI: 10.1080/03610926.2017.1303729

To link to this article: https://doi.org/10.1080/03610926.2017.1303729

Accepted author version posted online: 13

Mar 2017.

Published online: 08 Sep 2017.

Submit your article to this journal $\sqsubset$

Џ Article views: 57

Q View related articles $₫$

View Crossmark data $\asymp$

Full Terms \& Conditions of access and use can be found at

http://www.tandfonline.com/action/journallnformation?journalCode=Ista20 


\title{
Asymptotic estimate of variance with applications to stochastic differential equations arises in mathematical neuroscience
}

\author{
M. Rahman \\ Department of Mathematics \& Statistics, University of North Florida, Jacksonville, FL, USA
}

\begin{abstract}
Matrix representation of a limit of variance for circular process is given. It is shown that the variance is asymptotically measured by the decrease in spectral energy in one step of a Markov chain. Then we apply this result to a stochastic differential equation with parametric noise (which arises in mathematical neuroscience) and demonstrate how the results can be used to analyze propagation of a signal in sound mechanism.
\end{abstract}

ARTICLE HISTORY

Received 24 July 2015

Accepted 28 February 2017

\section{KEYWORDS}

Chain; circular Markov process; theta-dot network; variance; voltage control oscillator.

\section{MATHEMATICS SUBJECT CLASSIFICATION} 60J05; 60J10; 60J20; 60h35; $60 \mathrm{H} 10 ; 92 \mathrm{~B} 20 ; 97 \mathrm{M} 10$

\section{Introduction}

Approximation of stochastic differential equations (SDEs) with parametric noise plays an important role in a range of application areas, including engineering, mechanics, epidemiology, and neuroscience. A complete understanding of SDE theory with perturbed noise requires familiarity with advanced probability and stochastic processes. In this paper, we derive an asymptotic estimate of variance, and it is shown that numerical method gives a useful step toward solving SDEs with perturbed noise. Our goal is to diffuse the results to an audience not entirely familiar with functional notations or semi-group theory, but who might nonetheless be interested in the practical simulation of dynamical systems with fast noise or a slow manifold. A realization of a stochastic process $\theta$ determines a realization of the process

$$
\xi(t):=t^{-1 / 2} \int_{0}^{t} \varphi(\theta(s)) d s
$$

where $\varphi$ is a given real function. A discrete-time equivalent of (1.1) for a chain $\left\{\theta_{k}\right\}_{k \geq 0}$ whose state changes every unit time is

$$
\xi_{N}:=N^{-1 / 2} \sum_{n=0}^{N-1} \varphi\left(\theta_{n}\right)
$$

Expressions of form (1.1) or (1.2) arise in the approximation of SDEs with parametric noise. In this context, the asymptotic behavior of $\xi(t)$ as $t \rightarrow \infty$ or $\xi_{N}$ as $N \rightarrow \infty$ is of particular interest.

CONTACT M. Rahman mrahman@unf.edu E Department of Mathematics \& Statistics, University of North Florida, Jacksonville, FL-32224, USA.

Color versions of one or more of the figures in the article can be found online at www.tandfonline.com/lsta.

() 2017 Taylor \& Francis Group, LLC 
This work has been dedicated to this question in particular when $\theta$ is a discrete-space Markov chain/process. Most recent contributions have aimed in general at relaxing the assumptions on $\theta$ Skorokhod (2000), Skorokhod, Hoppensteadt, and Salehi (2002). Typically, an estimate of the form

$$
\begin{aligned}
\xi & \approx \mathcal{N}\left(0, \sigma^{2}\right) \\
t & \rightarrow \infty \text { or } \\
N & \rightarrow \infty
\end{aligned}
$$

is obtained, i.e., $\xi$ is asymptotically normally distributed with variance $\sigma^{2}$ depending on $\varphi$ and $\theta$. This result is known as a Functional Central Limit Theorem (FCLT).

We present in Sec. 2 an intuitive and self-contained proof for time-continuous processes (1.1), including a description of the fairly standard assumptions used (compact state space for $\theta$, homogeneity, and mixing). Our goal is to diffuse the results to an audience not entirely familiar with functional notations or semi-group theory, but who might nonetheless be interested in the practical simulation of dynamical systems with fast noise or a slow manifold. Section 3 covers similar ideas for discrete-time Markov chains (1.2).

We present in Sec. 4 a self contained proof for a limit of variance for discrete-space Markov chains as an extension of the recently published paper Rahman and Welfert (2013). In Sec. 5, we study a model in mathematical neuroscience that has some explanation in Hoppensteadt and Izhikevich (2006), Hoppensteadt (1997). There is a deterministic basis for this model, and additive noise does not capture the separate variation parameters encountered. Therefore, we consider the problem with parametric noise. In Sec. 6, we investigate how noise can slow down the wave propagation in $\dot{\theta}$-network model arises in mathematical neuroscience.

\section{FCLT for Markov processes}

Let $\theta(t)$ be a (continuous-time) Markov process in a measurable space $(\Theta, d \Theta)$ with transition probability

$$
0 \leq P\left(s, t, \theta, d \theta^{\prime}\right) \equiv \mathcal{P}\left(\theta(t) \in d \theta^{\prime} \mid \theta(s)=\theta\right) \leq 1
$$

for $0 \leq s \leq t, \theta \in \Theta$ and $d \theta^{\prime} \in d \Theta$. In other words $P\left(s, t, \theta, d \theta^{\prime}\right)$ is the probability that $\theta(t)$ is in the subset $d \theta^{\prime}$ when "started" at $\theta(s)=\theta$.

\subsection{Assumptions}

A1 $\theta(t)$ is homogeneous (or stationary), i.e.,

$$
P\left(s, t, \theta, d \theta^{\prime}\right)=P\left(0, t-s, \theta, d \theta^{\prime}\right)
$$

for any $0 \leq s \leq t$.

In the following, we write $P\left(t-s, \theta, d \theta^{\prime}\right)$ for $P\left(0, t-s, \theta, d \theta^{\prime}\right)$. We also require that

$$
\lim _{t \rightarrow 0} P\left(t, \theta, d \theta^{\prime}\right)=P\left(0, \theta, d \theta^{\prime}\right)=\chi\left(\theta, d \theta^{\prime}\right)= \begin{cases}1 & \text { if } \theta \in d \theta^{\prime} \\ 0 & \text { otherwise }\end{cases}
$$

The expected value of a bounded function $\varphi(\theta(t))$ with $\theta(s)=\theta$ for some $s \leq t$ is

$$
E_{\theta(s)=\theta}[\varphi(\theta(t))] \equiv \int_{\Theta} \varphi\left(\theta^{\prime}\right) P\left(s, t, \theta, d \theta^{\prime}\right)=\int_{\Theta} \varphi\left(\theta^{\prime}\right) P\left(t-s, \theta, d \theta^{\prime}\right)
$$


A consequence of $[\mathrm{A} 1]$ is

$$
E_{\theta(s)=\theta}[\varphi(\theta(t))]=E_{\theta(0)=\theta}[\varphi(\theta(t-s))]
$$

The relation $E_{\theta(t)=\theta}[\varphi(\theta(t))]=\varphi(\theta)$ for any $t \geq 0$ follows from (2.6). Taking $\varphi(\theta)=$ 1 for all $\theta \in \Theta$ in (2.7) yields the stochastic property

$$
\int_{\Theta} P\left(t, \theta, d \theta^{\prime}\right)=E_{\theta(0)=\theta}[1]=1
$$

for any $\theta$.

A2 $\theta(\cdot)$ has the Markov property (Borodin and Salminen, 2002, p. 6)

$$
E_{\theta(0)}[\varphi(\theta(s)) \psi(\theta(t))]=E_{\theta(0)}\left[\varphi(\theta(s)) E_{\theta(s)}[\psi(\theta(t))]\right]
$$

for all $0 \leq s \leq t$ and all bounded function $\varphi$ and $\psi$ If $\varphi=1_{\Theta}(2.10)$ reduces to

$$
E_{\theta(0)}[\psi(\theta(t))]=E_{\theta(0)}\left[E_{\theta(s)}[\psi(\theta(t))]\right]
$$

Another form of (2.11) can be obtained by substituting (2.7):

$$
\begin{aligned}
\int_{\Theta} \psi\left(\theta^{\prime \prime}\right) P\left(t, \theta, d \theta^{\prime \prime}\right) & =\int_{\Theta} E_{\theta(s)=\theta^{\prime}}[\psi(\theta(t))] P\left(s, \theta, d \theta^{\prime}\right) \\
& =\int_{\Theta} \int_{\Theta} \psi\left(\theta^{\prime \prime}\right) P\left(t-s, \theta^{\prime}, d \theta^{\prime \prime}\right) P\left(s, \theta, d \theta^{\prime}\right),
\end{aligned}
$$

which reduces to the Chapman-Lomonosov equation (Borodin and Salminen, 2002, p. 3)

$$
P\left(t, \theta, d \theta^{\prime \prime}\right)=\int_{\Theta} P\left(t-s, \theta^{\prime}, d \theta^{\prime \prime}\right) P\left(s, \theta, d \theta^{\prime}\right)
$$

valid for any $0 \leq s \leq t$. The relation (2.13) is illustrated in Figure 1. It also implies

$$
\begin{aligned}
& \left(\partial_{t} P\right)\left(u, \theta, d \theta^{\prime}\right) \underset{\Delta t \rightarrow 0}{\approx}(\Delta t)^{-1}\left(P\left(u+\Delta t, \theta, d \theta^{\prime}\right)-P\left(u, \theta, d \theta^{\prime}\right)\right) \\
& \underset{\Delta t \rightarrow 0}{=}(\Delta t)^{-1} \int_{\Theta} P\left(u, \theta, d \theta^{\prime \prime}\right)\left(P\left(\Delta t, \theta^{\prime \prime}, d \theta^{\prime}\right)-P\left(0, \theta^{\prime \prime}, d \theta^{\prime}\right)\right) \\
& \underset{\Delta t \rightarrow 0}{\approx} \int_{\Theta} P\left(u, \theta, d \theta^{\prime \prime}\right)\left(\partial_{t} P\right)\left(0, \theta^{\prime \prime}, d \theta^{\prime}\right) \\
& \underset{\Delta t \rightarrow 0}{=} \int_{\Theta} P\left(u, \theta, d \theta^{\prime \prime}\right) Q\left(\theta^{\prime \prime}, d \theta^{\prime}\right) \\
& \theta(0)=\theta
\end{aligned}
$$

Figure 1. Sample paths $\theta(t)$ originating from $\theta(0)=\theta$ and landing in $d \theta^{\prime \prime}$ at time $t$. Each path passes through a subset $d \theta^{\prime}$ of $\Theta$ at time $s$. 
using (2.6) and defining the infinitesimal generator

$$
Q\left(\theta, d \theta^{\prime}\right) \equiv\left(\partial_{t} P\right)\left(0, \theta, d \theta^{\prime}\right)
$$

The differential Equation (2.14) and initial condition (2.6) define $P\left(t, \theta, d \theta^{\prime}\right)$ for all $t>0$ and any $\theta \in \Theta$ and $d \theta^{\prime} \in d \Theta$.

A3 $\theta(t)$ satisfies

$$
E_{\theta(0)=\theta}\left[t^{-1} \int_{0}^{t} \varphi(\theta(s)) d s\right] \approx t^{-1} \int_{0}^{t} E_{\theta(0)=\theta}[\varphi(\theta(s))] d s
$$

as $t \rightarrow \infty$, for all bounded functions $\varphi$, uniformly in $\theta=\theta(0)$

A4 $\rho\left(d \theta^{\prime}\right) \equiv \lim _{t \rightarrow \infty} t^{-1} \int_{0}^{t} P\left(s, \theta, d \theta^{\prime}\right) d s$ exists uniformly in $\theta(0)=\theta$ [A3] and [A4] imply

$$
\begin{aligned}
\lim _{t \rightarrow \infty} t^{-1} \int_{0}^{t} E_{\theta(0)=\theta}[\varphi(\theta(s))] d s & =\lim _{t \rightarrow \infty} \int_{\Theta} \varphi\left(\theta^{\prime}\right) t^{-1} \int_{0}^{t} P\left(s, \theta, d \theta^{\prime}\right) d s \\
& =\int_{\Theta} \varphi\left(\theta^{\prime}\right) \rho\left(d \theta^{\prime}\right)
\end{aligned}
$$

By (2.17) condition (2.16) can therefore be reformulated as the ergodic property

$$
\lim _{t \rightarrow \infty} E_{\theta(0)=\theta}\left[t^{-1} \int_{0}^{t} \varphi(\theta(s)) d s\right]=\int_{\Theta} \varphi\left(\theta^{\prime}\right) \rho\left(d \theta^{\prime}\right),
$$

i.e., the expected long time average is $\varphi(\theta(t))$ is the ensemble average. Letting $s \rightarrow \infty$ with $t-s=u$ fixed in (2.12) shows that

$$
\int_{\Theta} \psi\left(\theta^{\prime \prime}\right) \rho\left(d \theta^{\prime \prime}\right)=\int_{\Theta} \psi\left(\theta^{\prime \prime}\right) \int_{\Theta} P\left(u, \theta^{\prime}, d \theta^{\prime \prime}\right) \rho\left(d \theta^{\prime}\right)
$$

for any $u \geq 0$. Note that [A4] holds if

$$
\rho\left(d \theta^{\prime}\right)=\lim _{t \rightarrow \infty} P\left(t, \theta, d \theta^{\prime}\right)
$$

exists uniformly in $\theta$ (Cesaro mean theorem). The strong form of (2.19) then reads

$$
\rho\left(d \theta^{\prime \prime}\right)=\int_{\Theta} P\left(u, \theta^{\prime}, d \theta^{\prime \prime}\right) \rho\left(d \theta^{\prime}\right)
$$

A5 The integral

$$
\mathcal{R} \varphi(\theta) \equiv \int_{0}^{\infty} E_{\theta(0)=\theta}[\varphi(\theta(u))] d u
$$

converges uniformly in $\theta(0)=\theta$.

From (2.17) a necessary condition for (2.22) to converge is

$$
\int_{\Theta} \varphi\left(\theta^{\prime}\right) \rho\left(d \theta^{\prime}\right)=0
$$

\subsection{FCLT}

Theorem 2.1. If [A1, A2, A3, A4, A5] hold for $\theta$ and $\varphi$ satisfies the zero average condition (2.23) then the function $\xi(t)$ from (1.1) satisfies

$$
\xi(t) \underset{t \rightarrow \infty}{\approx} \mathcal{N}\left(0, \sigma^{2}\right), \quad \sigma^{2}=2 \int_{\Theta} \varphi(\theta) \mathcal{R} \varphi(\theta) \rho(d \theta)
$$


Proof. Note that $\mathcal{R} \varphi(\theta)$ can also be written

$$
\begin{aligned}
\mathcal{R} \varphi(\theta) & =\int_{0}^{\infty} E_{\theta(0)=\theta}[\varphi(\theta(u+s-s))] d u \\
& =\int_{0}^{\infty} E_{\theta(s)=\theta}[\varphi(\theta(u+s))] d u \quad(\text { by }(2.8), \text { i.e., }[A 1]) \\
& =\int_{s}^{\infty} E_{\theta(s)=\theta}[\varphi(\theta(u))] d u \quad \text { (chang of variable) }
\end{aligned}
$$

for any $s \geq 0$. We have

$$
\begin{aligned}
E[\xi(t))] & =t^{-1 / 2} \int_{0}^{t} E_{\theta(0)=\theta}[\varphi(\theta(s))] d s \quad(\text { by }[\mathrm{A} 3]) \\
& \approx t^{-1 / 2} \mathcal{R}_{\omega} \varphi(\theta) \rightarrow 0 \quad(\text { by }[\mathrm{A} 5])
\end{aligned}
$$

as $t \rightarrow \infty$. On the other hand

$$
\begin{aligned}
E\left[\xi^{2}(t)\right] & =E_{\theta(0)=\theta}\left[t^{-1} \int_{0}^{t} \varphi(\theta(s)) \int_{0}^{t} \varphi(\theta(u)) d u d s\right] \\
& =2 E_{\theta(0)=\theta}\left[t^{-1} \int_{0}^{t} \varphi(\theta(s)) \int_{s}^{t} \varphi(\theta(u)) d u\right] d s \\
& \approx 2 t^{-1} \int_{0}^{t} E_{\theta(0)=\theta}\left[\varphi(\theta(s)) \int_{s}^{t} \varphi(\theta(u)) d u\right] d s \quad \text { (by [A5]) } \\
& =2 \int_{0}^{t} E_{\theta(0)=\theta}\left[\varphi(y(s)) E_{\theta(s)}\left[t^{-1} \int_{s}^{t} \varphi(y(u)) d u\right] d s \quad\right. \text { (by [A2]) } \\
& \approx 2 t^{-1} \int_{0}^{t} E_{\theta(0)=\theta}\left[\varphi(\theta(s)) \int_{s}^{t} E_{\theta(s)}[\varphi(\theta(u))] d u\right] d s \quad \text { (by [A5]) } \\
& \approx 2 t^{-1} \int_{0}^{t} E_{\theta(0)=\theta}\left[\varphi(\theta(s)) \int_{s}^{\infty} E_{\theta(s)}[\varphi(\theta(u))] d u\right] d s \\
& =2 t^{-1} \int_{0}^{t} E_{\theta(0)=\theta}[\varphi(\theta(s)) \mathcal{R} \varphi(\theta(s))] d s \quad \text { (by [A4]) } \\
& \approx 2 \int_{\Theta} \varphi(\theta) \mathcal{R} \varphi(\theta) \rho(d \theta) \quad(\text { by [A3], (2.17)) }
\end{aligned}
$$

as $t \rightarrow \infty$. The limit $\int_{s}^{t} \rightarrow \int_{s}^{\infty}$ is justified by the fact that

$$
\lim _{t \rightarrow \infty} f(t, g(t))=\lim _{t \rightarrow \infty} f\left(t, \lim _{t \rightarrow \infty} g(t)\right)
$$

for continuous functions $f$ and $g$ provided all limits exist.

\section{FCLT for Markov chains}

We now turn to discrete-time Markov processes, i.e., chains. For simplicity we assume that $\theta$ changes at regular time intervals.

\section{1. $F C L T$}

First, we assume that updates are made every unit time, i.e.,

$$
\theta(t)=\theta_{\lfloor t\rfloor}
$$


Then

$$
\xi(N)=N^{-1 / 2} \sum_{n=0}^{N-1} \varphi\left(\theta_{n}\right)
$$

Define

$$
\mathcal{R}^{1,0} \varphi(\theta) \equiv \sum_{n \geq 0} E_{\theta_{0}=\theta}\left[\varphi\left(\theta_{n}\right)\right]
$$

and

$$
\mathcal{R}^{1,1} \varphi(\theta) \equiv \sum_{n \geq 1} E_{\theta_{0}=\theta}\left[\varphi\left(\theta_{n}\right)\right]
$$

Observe that

$$
\mathcal{R}^{1,0} \varphi(\theta)=E_{\theta_{0}=\theta}\left[\varphi\left(\theta_{0}\right)\right]+\mathcal{R}^{1,1} \varphi(\theta)=\varphi(\theta)+\mathcal{R}^{1,1} \varphi(\theta)
$$

Integrals appearing in Sec. 2 can be straightforwardly replaced by discrete sums except in (2.26). This is because in the folding process: square $0 \leq s, u \leq t \rightarrow$ triangle $0 \leq s \leq u \leq t$, the line $s=u$ is counted twice in the discrete case, so one occurrence must be subtracted. Specifically,

$$
\begin{aligned}
E\left[\xi^{2}(N)\right] & =E_{\theta_{0}=\theta}\left[N^{-1} \sum_{n=0}^{N-1} \varphi\left(\theta_{n}\right) \sum_{p=0}^{N-1} \varphi\left(\theta_{p}\right)\right] \\
& =2 E_{\theta_{0}=\theta}\left[N^{-1} \sum_{n=0}^{N-1} \varphi\left(\theta_{n}\right) \sum_{p=n}^{N-1} \varphi\left(\theta_{p}\right)\right]-E_{\theta_{0}=\theta}\left[N^{-1} \sum_{n=0}^{N-1} \varphi^{2}\left(\theta_{n}\right)\right]
\end{aligned}
$$

The first term is the discrete analog of (2.27) and asymptotically converges to (2.28), while the second term converges to

$$
\int_{\Theta} \varphi^{2}(\theta) \rho(d \theta)
$$

according to (2.18). Since $\xi(t) \approx \xi(N)$ as $N=\lfloor t\rfloor \rightarrow \infty$, this shows

$$
E\left[\xi^{2}(t)\right] \approx 2 \int_{\Theta} \varphi(\theta) \mathcal{R}^{1,0} \varphi(\theta) \rho(d \theta)-\int_{\Theta} \varphi^{2}(\theta) \rho(d \theta)
$$

as $t \rightarrow \infty$. The relation (3.32) yields the equivalent form

$$
E\left[\xi^{2}(t)\right] \approx 2 \int_{\Theta} \varphi(\theta) \mathcal{R}^{1,1} \varphi(\theta) \rho(d \theta)+\int_{\Theta} \varphi^{2}(\theta) \rho(d \theta)
$$

To obtain a similar result when updates are made every $\Delta t>0$, i.e.,

$$
\theta(t)=\theta_{\lfloor t / \Delta t\rfloor}
$$

consider the random process

$$
\theta_{\Delta t}(t) \equiv \theta(t \Delta t)
$$


and define

$$
\begin{aligned}
\xi_{\Delta t}(t) & \equiv t^{-1 / 2} \int_{0}^{t} \varphi\left(\theta_{\Delta t}(s)\right) d s \\
& =(\Delta t)^{-1} t^{-1 / 2} \int_{0}^{t \Delta t} \varphi(\theta(u)) d u \\
& =(\Delta t)^{-1 / 2} \xi(t \Delta t)
\end{aligned}
$$

and (using $\left.\theta_{\Delta t}(0)=\theta(0)\right)$

$$
\begin{aligned}
\mathcal{R}^{\Delta t, 0} \varphi(\theta) & \equiv \int_{0}^{\infty} E_{\theta(0)=\theta}[\varphi(\theta(s))] d s \\
& =\Delta t \int_{0}^{\infty} E_{\theta(0)=\theta}[\varphi(\theta(u \Delta t))] d u \\
& =\Delta t \int_{0}^{\infty} E_{\theta_{\Delta t}(0)=\theta}\left[\varphi\left(\theta_{\Delta t}(u)\right)\right] d u \\
& =\Delta t \mathcal{R}^{1,0} \varphi(\theta)
\end{aligned}
$$

Because $\theta_{\Delta t}(t)=\theta_{\lfloor t\rfloor}$, we can apply (3.34) to $\theta_{\Delta t}(t)$ and $\xi_{\Delta t}(t)$. Note that the space $\Theta_{\Delta t}$ of possible outcomes of $\theta_{\Delta t}$ is equal to $\Theta$ and that $\rho_{\Delta t}\left(d \theta_{\Delta t}\right)=\rho(d \theta)$. From (3.36) and (3.37) we obtain, as $N \rightarrow \infty$

$$
E[\xi(N \Delta t)]=(\Delta t)^{1 / 2} E\left[\xi_{\Delta t}(N)\right] \approx 0
$$

and

$$
\begin{aligned}
E\left[\xi^{2}(N \Delta t)\right]= & \Delta t E\left[\xi_{\Delta t}^{2}(N)\right] \quad(\text { by }(3.36)) \\
\approx & 2 \Delta t \int_{\Theta \Delta t} \varphi\left(\theta_{\Delta t}\right) \mathcal{R}^{1,0} \varphi\left(\theta_{\Delta t}\right) \rho_{\Delta t}\left(d \theta_{\Delta t}\right) \\
& -\Delta t \int_{\Theta \Delta} \varphi^{2}\left(\theta_{\Delta t}\right) \rho_{\Delta t}\left(d \theta_{\Delta t}\right) \quad(\text { by }(3.33)) \\
= & 2 \int_{\Theta} \varphi(\theta) \mathcal{R}^{\Delta t, 0} \varphi(\theta) \rho(d \theta)-\Delta t \int_{\Theta} \varphi^{2}(\theta) \rho(d \theta) \quad \text { (by (3.37)) } \\
= & 2 \int_{\Theta} \varphi(\theta) \mathcal{R}^{\Delta t, 1} \varphi(\theta) \rho(d \theta)+\Delta t \int_{\Theta} \varphi^{2}(\theta) \rho(d \theta)
\end{aligned}
$$

using

$$
\mathcal{R}^{\Delta t, 1} \varphi(\theta) \equiv \mathcal{R}^{\Delta t, 0} \varphi(\theta)-\Delta t \varphi(\theta)=\Delta t \sum_{n \geq 1} E_{\theta_{0}=\theta}\left[\varphi\left(\theta_{n}\right)\right]
$$

\section{FCLT for discrete-space Markov chains}

We now turn to the case where $\Theta=\left\{\theta_{1}, \ldots, \theta_{n}\right\}$ and let

$$
\underline{\theta}=\left[\begin{array}{c}
\theta_{1} \\
\vdots \\
\theta_{n}
\end{array}\right], \quad \varphi(\underline{\theta})=\left[\begin{array}{c}
\varphi\left(\theta_{1}\right) \\
\vdots \\
\varphi\left(\theta_{n}\right)
\end{array}\right], \quad \text { and } \quad \underline{e}=\left[\begin{array}{c}
1 \\
\vdots \\
1
\end{array}\right]
$$

The transition probability $P\left(\Delta t, \theta, d \theta^{\prime}\right)$ can be represented by an $n \times n$ matrix $P$ such that $P_{i, j}=P\left(\Delta t, \theta_{i}, \theta_{j}\right)$ denotes the probability that $\theta(\Delta t)=\theta_{j}$ if $\theta(0)=\theta_{i}(P$ depends on $\Delta t$, 
but this dependence is omitted from the notation for simplicity). The matrix $P$ satisfies the following properties.

- $P$ is non negative (its entries are probabilities).

- $P$ is stochastic; i.e., $P \underline{e}=\underline{e}$ (one of the $\theta_{j}$ must be the outcome of a transition from $\theta_{i}$ ). Then $\lambda_{1}=1$ is an eigenvalue of $P$ and $1 \leq \rho(P) \leq\|P\|_{\infty}=1$ shows that all other eigenvalues have modulus at most 1 . We shall assume that

- $P$ is irreducible, i.e., any state $\theta_{j}$ can be eventually be reached in a finite number of steps with a non zero probability (this is the case if $P>0$ ). This implies that $\lambda_{1}=1=\rho(P)$ has multiplicity one (e.g., see Berman and Plemmons 1994).

- $P$ is aperiodic (or acyclic, e.g., not a permutation). This implies that $\left|\lambda_{j}\right|<1$ for $j=$ $2, \ldots, n$ (e.g., see Karlin 1966; the chain is then called regular).

The above conditions guarantee the existence of a unique vector $\underline{v}>0$ such that

$$
\underline{v}^{T} \underline{e}=1
$$

and $\lim _{N \rightarrow \infty} P^{N}=\underline{e v}^{T}$. The vector $\underline{v}$ is the unique positive left eigenvector associated to $\lambda_{1}=$ 1 satisfying (4.39). It is natural to consider the (spectral) decomposition

$$
P=\underline{e v}^{T}+S
$$

where

$$
\underline{v}^{T} S=\underline{0}^{T}, \quad S \underline{e}=\underline{0}
$$

and $\rho(S)<1$. From Chapman-Kolmogorov equation and the homogeneity property (see, Rahman and Welfert 2013), we have

$$
P\left(2 \Delta t, \theta_{i}, \theta_{j}\right)=\sum_{k=1}^{n} P\left(\Delta t, \theta_{i}, \theta_{k}\right) P\left(\Delta t, \theta_{k}, \theta_{j}\right)=\sum_{k=1}^{n} P_{i, k} P_{k, j}=P_{i, j}^{2},
$$

and, by induction

$$
P\left(N \Delta t, y_{i}, y_{j}\right)=P_{i, j}^{N}=\left(\underline{e v}^{T}+S^{N}\right)_{i, j} \approx\left(\underline{e v}^{T}\right)_{i, j}=v_{j}
$$

as $N \rightarrow \infty$, independently of $i$ (i.e., $\theta_{i}$ ). Thus

$$
\underline{v}=\rho_{\Delta t}(\underline{\theta})
$$

defines the limit distribution from condition [A3]. An explicit expression of the coefficients of $\underline{v}$ in terms of the coefficients of $P$ can be found in (Romanovsky 1970, p. 21).

The relation the expected value yields

$$
E_{\theta(0)=\theta_{j}}[\varphi(\theta(N \Delta t))]=\sum_{j=1}^{n} \varphi\left(\theta_{j}\right) P\left(N \Delta t, \theta_{j}, \theta_{i}\right)=\sum_{j=1}^{n}\left(P^{N}\right)_{i, j} \varphi\left(\theta_{j}\right)=\left(P^{N} \varphi(\underline{\theta})\right)_{j}
$$
i.e.,

$$
E_{\theta(0)=\underline{\theta}}[\varphi(\theta(N \Delta t))]=\left[\begin{array}{c}
E_{\theta(0)=\theta_{1}}[\varphi(\theta(N \Delta t))] \\
\vdots \\
E_{\theta(0)=\theta_{n}}[\varphi(\theta(N \Delta t))]
\end{array}\right]=P^{N} \varphi(\underline{\theta})
$$

The zero average condition on $\varphi$ becomes

$$
0=\int_{\Theta} \varphi(\theta) \rho(d \theta)=\sum_{j=1}^{n} \varphi\left(\theta_{j}\right) \rho\left(\theta_{j}\right)=\rho(\underline{\theta})^{T} \varphi(\underline{\theta})=\underline{v}^{T} \varphi(\underline{\theta})
$$


The relation (4.44) implies $P \varphi(\mathbf{y})=\varphi(\mathbf{y})$. Therefore

$$
\begin{aligned}
\mathcal{R}^{\Delta t, 1} \varphi(\underline{\theta}) & =\Delta t \sum_{N=1}^{\infty} E_{\theta(0)=\underline{\theta}}[\varphi(\theta(N \Delta t))] \\
& =\Delta t \sum_{N=1}^{\infty} P^{N} \varphi(\underline{\theta}) \\
& =\Delta t \sum_{N=1}^{\infty} S^{N} \varphi(\underline{\theta}) \\
& =\Delta t(I-S)^{-1} S \varphi(\underline{\theta})
\end{aligned}
$$

Note that (4.45) implies

$$
\mathcal{R}^{\Delta t, 1} \varphi(\underline{\theta})-P \mathcal{R}^{\Delta t, 1} \varphi(\underline{\theta})=\Delta t P \varphi(\underline{\theta})
$$

Because of (4.41) we also have

$$
\underline{v}^{T} \mathcal{R}^{\Delta t, 1} \varphi(\underline{\theta})=0
$$

With $V=\operatorname{diag}(\underline{v})$ we obtain

$$
\begin{aligned}
\sigma_{\Delta t}^{2} & =2 \sum_{j=1}^{n} \varphi\left(\theta_{j}\right) \rho\left(\theta_{j}\right)\left(\mathcal{R}^{\Delta t, 1} \varphi(\underline{\theta})\right)_{j}+\Delta t \sum_{j=1}^{n} \varphi^{2}\left(\theta_{j}\right) \rho\left(\theta_{j}\right) \\
& =2 \Delta t \varphi(\underline{\theta})^{T} V(I-S)^{-1} S \varphi(\underline{\theta})+\Delta t \varphi(\underline{\theta})^{T} V \varphi(\underline{\theta}) \\
& =\Delta t \varphi(\underline{\theta})^{T} V(I-S)^{-1}(I+S) \varphi(\underline{\theta})
\end{aligned}
$$

as $t \rightarrow \infty$. Expression (4.49) represents the limit of a variance and is thus expected to be non negative. A direct algebraic proof is given in the following.

Let $P$ satisfy the conditions given at the beginning of this section. Recall that the limit distribution $\underline{v}>\underline{0}$, so that the matrix $V=\operatorname{diag}(\underline{v})$ is (diagonal) positive definite. Define the norm $\|\cdot\|_{V}$ such that $\|\underline{u}\|_{V}^{2}=\underline{u}^{T} V \underline{u}$ for $\underline{u} \in \underline{R}^{n}$.

Lemma 4.1. $\|P \underline{u}\|_{V} \leq\|\underline{u}\|_{V}$ for any $\underline{u} \in \mathbb{R}^{n}$.

Proof. Let $\|\cdot\|_{V}$ also denote the spectral norm induced by $\|\cdot\|_{V}$ and $\rho(A)$ the spectral radius of a matrix $A$. Then

$$
\begin{aligned}
\|P\|_{V}^{2} & =\left\|V^{\frac{1}{2}} P\right\|_{2}^{2} \\
& =\rho\left(\left(V^{\frac{1}{2}} P\right)^{T} V^{\frac{1}{2}} P\right) \\
& =\rho\left(P^{T} V P\right) \\
& \leq\left\|P^{T} V P\right\|_{\infty} \\
& =\max _{1 \leq i \leq n}\left(P^{T} V P \underline{e}\right)_{i} \quad(\text { since } P, V \geq 0) \\
& =\max _{1 \leq i \leq n}\left(P^{T} V \underline{e}\right)_{i} \quad(\text { since } P \underline{e}=\underline{e}) \\
& =\max _{1 \leq i \leq n}\left(P^{T} \underline{v}\right)_{i} \\
& =\max _{1 \leq i \leq n} v_{i} \quad\left(\text { since } P^{T} \underline{v}=\underline{v}\right) \\
& \leq 1
\end{aligned}
$$


Thus

$$
\|P \underline{u}\|_{V} \leq\|P\|_{V}\|\underline{u}\|_{V} \leq\|\underline{u}\|_{V}
$$

Theorem 4.2. Let $\varphi(\underline{\theta}) \in \mathbb{R}^{n}$ be such that $\underline{v}^{T} \varphi(\underline{\theta})=0$. Then

1. $\varphi(\underline{\theta})=\frac{1}{\sqrt{\Delta t}}(I-S) \underline{u}$ with $\underline{u}=\frac{1}{\sqrt{\Delta t}} \mathcal{R}^{\Delta t, 0} \varphi(\underline{\theta})$

2. $\Delta t \varphi(\underline{\theta})^{T} V(I-S)^{-1}(I+S) \varphi(\underline{\theta})=\|\underline{u}\|_{V}^{2}-\|P \underline{u}\|_{V}^{2}$

Proof. From (4.47) we obtain

$$
\begin{aligned}
(I-S) \mathcal{R}^{\Delta t, 0} \varphi(\underline{\theta}) & =(I-S)\left(\Delta t \varphi(\underline{\theta})+\mathcal{R}^{\Delta t, 1}(\underline{\theta})\right) \\
& =\Delta t(I-S) \varphi(\underline{\theta})+\Delta t S \varphi(\underline{\theta}) \\
& =\Delta t \varphi(\underline{\theta})
\end{aligned}
$$

which shows 1 . From (4.44) and (4.48) we get

$$
\underline{v}^{T} \underline{u}=\frac{1}{\sqrt{\Delta t}} \underline{v}^{T} \mathcal{R}^{\Delta t, 0} \varphi(\underline{\theta})=\underline{v}^{T} \varphi(\underline{\theta})+\frac{1}{\sqrt{\Delta t}} \underline{v}^{T} \mathcal{R}^{\Delta t, 1} \varphi(\underline{\theta})=0
$$

Thus

$$
\begin{aligned}
\|S \underline{u}\|_{V}^{2} & =\underline{u}^{T} S^{T} V S \underline{u} \\
& =\underline{u}^{T}\left(P^{T}-\underline{v}^{T}\right) V\left(P-\underline{e v}^{T}\right) \underline{u} \\
& =\underline{u}^{T} P^{T} V P \underline{u} \\
& =\|P \underline{u}\|_{V}^{2}
\end{aligned}
$$

and

$$
\begin{aligned}
\Delta t \varphi(\underline{\theta})^{T} V(I-S)^{-1}(I+S) \varphi(\underline{\theta}) & =\underline{u}^{T}(I-S)^{T} V(I+S) \underline{u} \\
& =\underline{u}^{T} V \underline{u}-\underline{u}^{T} V S \underline{u}+\underline{u}^{T} S^{T} V \underline{u}-\underline{u}^{T} S^{T} V S \underline{u} \\
& =\|\underline{u}\|_{V}^{2}-\|S \underline{u}\|_{V}^{2} \\
& =\|\underline{u}\|_{V}^{2}-\|P \underline{u}\|_{V}^{2}
\end{aligned}
$$

From Lemma 4.1 and Theorem 4.2, the quantity $\Delta t \varphi(\underline{\theta})^{T} V(I-S)^{-1}(I+S) \varphi(\underline{\theta})$ is thus non negative. The vector

$$
\underline{u}=\frac{1}{\sqrt{\Delta t}} \sum_{N \geq 0} P^{N} \varphi(\underline{\theta})
$$

defined in Theorem 4.2 is an ensemble average approximation of $\xi(t)$ defined in (1.1). The variance of $\xi$ is thus asymptotically measured by the decrease in spectral energy in one step of the Markov chain.

\subsection{Example: Two-state Markov chain}

Let $n=2$ and

$$
P=P(\Delta t)=I+\Delta t Q, \quad Q=\left[\begin{array}{cc}
-a & a \\
b & -b
\end{array}\right]
$$

with $0<a, b<1,0<\Delta t<\min \left(\frac{1}{a}, \frac{1}{b}\right) \leq \frac{2}{a+b}$. Then (4.40) holds with

$$
\underline{v}=\frac{1}{a+b}\left[\begin{array}{l}
b \\
a
\end{array}\right] \text { and } S=\frac{1-\Delta t(a+b)}{a+b}\left[\begin{array}{cc}
a & -a \\
-b & b
\end{array}\right]
$$


As a result $V=\frac{1}{a+b}\left[\begin{array}{ll}b & \\ & \end{array}\right]$, and

$$
\Delta t V(I-S)^{-1}(I+S)=\frac{1}{(a+b)^{3}}\left[\begin{array}{cc}
b\left(2 a+\Delta t\left(b^{2}-a^{2}\right)\right) & -2 a b(1-\Delta t(a+b)) \\
-2 a b(1-\Delta t(a+b)) & a\left(2 b+\Delta t\left(a^{2}-b^{2}\right)\right)
\end{array}\right]
$$

is (symmetric) positive definite for any choice $0<a, b<1$ and $0<\Delta t<\frac{2}{a+b}$. A simplified expression for (4.49) is obtained using (4.44), i.e.,

$$
\varphi(\underline{\theta})=\left[\begin{array}{c}
1 \\
-\frac{b}{a}
\end{array}\right] \varphi\left(\theta_{1}\right)
$$

We obtain

$$
\begin{gathered}
\sigma_{\Delta t}^{2}=\frac{b(2-\Delta t(a+b))}{a(a+b)}\left(\varphi\left(\theta_{1}\right)\right)^{2} \\
=\frac{a b(2-\Delta t(a+b))}{(a+b)^{3}}\left(\varphi\left(\theta_{1}\right)-\varphi\left(\theta_{2}\right)\right)^{2}
\end{gathered}
$$

Remark 1. For the matrix $P$ in (4.50) a simple calculation shows that

$$
\mathcal{R}^{\Delta t, 0} \varphi(\underline{\theta})=\frac{1}{a+b} \varphi(\underline{\theta})
$$

is independent of $\Delta t$, while

$$
\mathcal{R}^{\Delta t, 1} \varphi(\underline{\theta})=\mathcal{R}^{\Delta t, 0} \varphi(\underline{\theta})-\Delta t \varphi(\underline{\theta})=\frac{1-\Delta t(a+b)}{a+b} \varphi(\underline{\theta})
$$

is not.

Remark 2. As $\Delta t \rightarrow 0$ the expression (4.53) becomes

$$
\sigma_{\Delta t}^{2}=\frac{2 a b}{(a+b)^{3}}\left(\varphi\left(\theta_{1}\right)-\varphi\left(\theta_{2}\right)\right)^{2}
$$

The same limit is valid if (4.50) is replaced by

$$
P(\Delta t)=I+\Delta t Q+o(\Delta t)
$$

with $\lim _{\Delta t \rightarrow 0} \frac{o(\Delta t)}{\Delta t}=0$, in particular if $P(\Delta t)=e^{\Delta t Q}$. In this case, $Q$ is the infinitesimal generator of the semi-group $P(\Delta t)$, which is defined for any $\Delta t>0$.

Remark 3. The range $0<\Delta t<\frac{2}{a+b}$ where the matrix (4.51) is positive definite corresponds to the interval of stability of Euler's method applied to the solution of the initial value problem $P^{\prime}(t)=Q P(t), P(0)=I$, with step size $\Delta t$, which yields (4.50) after the first step. Of course the matrix (4.50) ceases to be non negative before reaching the limit of stability.

\section{A model neural network}

In mathematical neuroscience, a signal of bursts can be described by its physical realization in terms of the membrane potentials, $v(t)$, or by its phase angle $\theta(t)$, where $v(t)=V(\theta(t))$ and $V$ is a fixed wave form (e.g., $V(\theta)=\cos \theta$ ).

If the tissue is of Hodgkin Type I (see, Hoppensteadt and Izhikevich 2006), then the Voltage Control Oscillator(VCO) provides a base for modelling:

$$
\dot{\theta}=\omega+\cos \theta
$$


When $0 \leq \omega \leq 1, \theta \rightarrow \cos ^{-1} \omega$ as $t \rightarrow \infty$. But if $\omega>1, \theta \rightarrow \infty$. If we are reading output of the system as a periodic function of $\theta$, then the output stabilizes in the first case, and it oscillates in the second. The switch in the system's behavior results from the saddle-node bifurcation that occurs when $\omega$ increases through the value of 1 .

Voltage-controlled oscillator (VCO) arises in mathematical neuroscience as a canonical model of excitable neural tissue of Type $I$ (see Hoppensteadt 1997). A network of $N$ such electronic circuits that are connected is described in Figure 2, where $\theta_{i}$ is the phase of $\mathrm{VCO}_{i}$ output, $\omega_{i}$ is the center frequency of $\mathrm{VCO}_{i}$, and $\mu_{i}$ is the sensitivity of $\mathrm{VCO}_{i}$, and we consider $\dot{\theta}_{i}=\omega_{i}+\mu_{i}\left(f\left(\theta_{i}\right)+V\left(\theta_{i-1}, \dot{\theta}_{i-1}\right)\right)$. Typically $\mu f(\theta)=\cos \theta, \quad \omega \simeq 1$. VCO is the model of saddle-node bifurcation on limit cycle (SNLC).

Consider the equation $\dot{x}=g(x)$ around $x=0$ s.t. $g^{\prime}(0)=0$. Then

$$
\dot{x} \simeq a+b x^{2}, \quad a=g(0), \quad b=\frac{1}{2} g^{\prime \prime}(0)
$$

Let $x=\tan \frac{\theta}{2}$. Then

$$
\dot{\theta} \simeq \omega+\mu \cos \theta, \quad \omega=a+b, \quad \mu=a-b
$$

If $|\omega|<|\mu|$, then the system has two static states (1 stable, 1 unstable). If $|\omega|>|\mu|$, then the system has no static state. Traditionally, $V(\theta, \dot{\theta})=V(\theta)=K \cos \theta$. Hoppensteadt (1997) proposed $V(\theta, \dot{\theta})=V(\dot{\theta})=K \dot{\theta} ;$; $\dot{\theta}$-network".

Following this approach, we consider a network of $N$ neurons using VCON's described by a phase variables $\theta_{j}(t), j=1, \ldots, N$ and the voltage output of each VCON is $\cos \theta_{j}(t)$. The connection in the network is defined by the matrix $C$ having dimensions $N \times N$. Using a synaptic input function $F(t)$, we write a neural networks in terms of their phases supposing that the output of a synapse is proportional to $\dot{\theta}$; that is, neuro transmitter is released in proportion to the number of action potentials arriving per unit time (see Hoppensteadt 1997; Hoppensteadt and Izhikevich 2006). With this notation, we have the following model:

$$
\left\{\begin{aligned}
\tau_{j} \dot{\theta}_{j}(t) & =\omega_{j}+\cos \theta_{j}(t)+F(t)+\mu \sum_{k=1}^{N} C_{j k} \dot{\theta}_{k}(t) \\
\theta_{j}(0) & =0
\end{aligned}\right.
$$

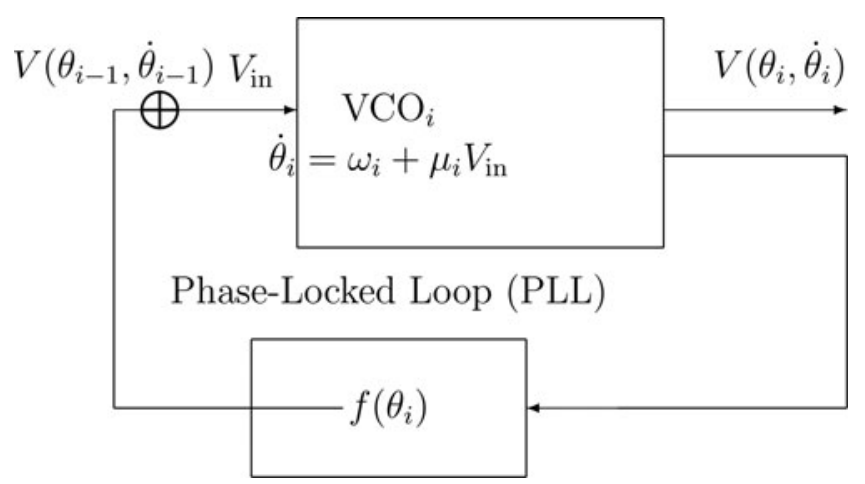

Figure 2. VCO as SNLC bifurcation $-\dot{\theta}$-network. 
where $\theta_{j}(t) \in \mathbb{R}$ is the phases, $\tau_{j}$ are time constants, $\mu$ is the strength of connection, and matrix $C$ is an $N \times N$ matrix of circular connection defined by

$$
C=\left(\begin{array}{cccccc}
0 & 1 & 0 & \ldots & \ldots & 0 \\
0 & 0 & 1 & \ldots & \ldots & 0 \\
\ldots & \ldots & \ldots & 1 & \ldots & 0 \\
\ldots & \ldots & \ldots & \ldots & \ldots & 1 \\
1 & 0 & 0 & 0 & \ldots & 0
\end{array}\right)
$$

Thus, using the matrix $C$ we can write (5.54) in matrix form

$$
\begin{cases}(\tau-\mu C) \dot{\theta}(t) & =\omega+F(t)+\cos \theta(t) \\ \theta_{j}(0) & =0\end{cases}
$$

Now if we use Markov chain (and Gaussian white) noise in the connection, then the matrix $C$ can be written as

$$
C(y(t / \epsilon))=\left(\begin{array}{ccccc}
0 & y_{1}(t / \epsilon) & 0 & \ldots & 0 \\
0 & 0 & y_{2}(t / \epsilon) & \ldots & 0 \\
\ldots & \ldots & \ldots & y_{3}(t / \epsilon) & 0 \\
\ldots & \ldots & \ldots & \ldots & y_{N-1}(t / \epsilon) \\
y_{N}(t / \epsilon) & 0 & 0 & 0 & 0
\end{array}\right)
$$

and then using $C(y(t / \epsilon))$ in (5.56), we obtain

$$
\begin{cases}(\tau-\mu C(y(t / \epsilon)) \dot{\theta}(t) & =\omega+F(t)+\cos \theta(t) \\ \theta(0) & =\end{cases}
$$

If the matrix $\tau-\mu C(y(t / \epsilon))$ is invertible, then we have

$$
\left\{\begin{aligned}
\dot{\theta} & =(\tau-\mu C(y(t / \epsilon)))^{-1}(\omega+F(t)+\cos \theta(t)) \equiv f(t, \epsilon, \omega, \theta, y) \\
\theta(0) & =0
\end{aligned}\right.
$$

Using limiting distribution $\rho$ and states $y_{1}$ and, $y_{2}$, we have the following average model:

$$
\begin{aligned}
\dot{\bar{\theta}}(t)= & \rho_{1}\left(\tau-\mu C\left(y_{1}\right)\right)^{-1}(\omega+F(t)+\cos \bar{\theta}(t)) \\
& +\rho_{2}\left(\tau-\mu C\left(y_{2}\right)\right)^{-1}(\omega+F(t)+\cos \bar{\theta}(t)) \\
\equiv & \bar{f}(t, \epsilon, \omega, \theta)
\end{aligned}
$$

and the stochastic correction equation (see Rahman, Jackiewicz, and Welfert 2007) can be written as

$$
d \tilde{\theta}(t)=\bar{f}_{\theta}(t, \omega, \bar{\theta}) \tilde{\theta}(t)+\sigma(t) d W
$$

where $\bar{f}_{\theta}(t, \omega, \bar{\theta})$ will be computed using (5.58) and $\rho$ and $d W=\sqrt{d t} N(0,1)$ with $W(0)=$ 0 . Now using limiting distribution $\rho_{1}$ and $\rho_{2}$, we can write $\bar{f}(t, \omega, \bar{\theta})$ in the form

$$
\begin{aligned}
\bar{f}(t, \omega, \bar{\theta})= & \rho_{1}\left(\tau-\mu C\left(y_{1}\right)\right)^{-1}(\omega+F(t)+\cos \bar{\theta}) \\
& +\rho_{2}\left(\tau-\mu C\left(y_{2}\right)\right)^{-1}(\omega+F(t)+\cos \bar{\theta}) \\
= & \left(\left(\tau-\mu C\left(y_{1}\right)\right)^{-1} \rho_{2}\left(\tau-\mu C\left(y_{2}\right)\right)^{-1}\right) \\
& \times(\omega+F(t)+\cos \bar{\theta}(t))
\end{aligned}
$$

Now, evaluating $f(t, \omega, \bar{\theta}, y)$ at $y_{1}$ and $y_{2}$, we have

$$
f\left(t, \omega, \bar{\theta}, y_{1}\right)=\left(\tau-\mu C\left(y_{1}\right)\right)^{-1}(\omega+F(t)+\cos \bar{\theta})
$$


and

$$
f\left(t, \omega, \bar{\theta}, y_{2}\right)=\left(\tau-\mu C\left(y_{2}\right)\right)^{-1}(\omega+F(t)+\cos \bar{\theta})
$$

Therefore, we have $\varphi\left(y_{1}\right)$ and $\varphi\left(y_{2}\right)$ such that $\rho_{1} \varphi\left(y_{1}\right)+\rho_{2} \varphi\left(y_{2}\right)=0$ (zero average condition for $\varphi$ ), where

$$
\begin{aligned}
\varphi\left(y_{1}\right)= & \left(\tau-\mu C\left(y_{1}\right)\right)^{-1}(\omega+F(t)+\cos \bar{\theta}(t)) \\
& -\rho_{1}\left(\tau-\mu C\left(y_{1}\right)\right)^{-1}(\omega+F(t)+\cos \bar{\theta}(t)) \\
& -\rho_{2}\left(\tau-\mu C\left(y_{2}\right)\right)^{-1}(\omega+\cos \bar{\theta}(t)) \\
= & \left(\tau-\mu C\left(y_{1}\right)\right)^{-1}(\omega+F(t)+\cos \theta(t))\left(1-\rho_{1}\right) \\
& -\left(\tau-\mu C\left(y_{2}\right)\right)^{-1}(\omega+F(t)+\cos \theta(t)) \rho_{2} \\
= & \left(\tau-\mu C\left(y_{1}\right)\right)^{-1}(\omega+F(t)+\cos \theta(t)) \rho_{2} \\
& -\left(\tau-\mu C\left(y_{2}\right)\right)^{-1}(\omega+F(t)+\cos \theta(t)) \rho_{2} \\
= & {\left[\left(\tau-\mu C\left(y_{1}\right)\right)^{-1}-\left(\tau-\mu C\left(y_{2}\right)\right)^{-1}\right] \rho_{2} } \\
& \times(\omega+F(t)+\cos \theta(t))
\end{aligned}
$$

and

$$
\begin{aligned}
\varphi\left(y_{2}\right)= & \left(\tau-\mu C\left(y_{2}\right)\right)^{-1}(\omega+F(t)+\cos \bar{\theta}(t)) \\
& -\rho_{1}\left(\tau-\mu C\left(y_{1}\right)\right)^{-1}(\omega+F(t)+\cos \bar{\theta}(t)) \\
& -\rho_{2}\left(\tau-\mu C\left(y_{2}\right)\right)^{-1}(\omega+F(t)+\cos \bar{\theta}) \\
= & {\left.\left[\left(\tau-\mu C\left(y_{2}\right)\right)^{-1} \rho_{2}-\left(\tau-\mu C\left(y_{1}\right)\right)^{-1}\right) \rho_{1}\right] } \\
& \times(\omega+F(t)+\cos \theta(t))
\end{aligned}
$$

Then using two-state Markov processes, we find that the following expressions for variance of the stochastic differential system (5.1) can be written as

$$
\begin{aligned}
\sigma^{2}(t)= & \frac{b}{a(a+b)}(2-\Delta t(a+b)) \varphi\left(y_{1}\right)^{2} \\
= & \frac{b}{a(a+b)}(2-\Delta t(a+b)) \\
& \times\left[\left(\left(\tau-\mu C\left(y_{1}\right)\right)^{-1}-\rho_{2}\left(\tau-\mu C\left(y_{2}\right)\right)^{-1}\right)\right. \\
& \times(\omega+F(t)+\cos \theta(t))]^{2} \\
= & \frac{b}{a(a+b)}(2-\Delta t(a+b))\left[\left(\left(\tau-\mu C\left(y_{1}\right)\right)^{-1}\right.\right. \\
& \left.\left.-\rho_{2}\left(\tau-\mu C\left(y_{2}\right)\right)^{-1}\right)(\omega+F(t)+\cos \theta(t))\right]^{2} .
\end{aligned}
$$

Using Equation (3.14), we have

$$
f_{\theta}(\bar{\theta}(t))=(\tau-\mu C(y / \epsilon))^{-1}(-\sin \theta(t))
$$

and then using the limiting distribution $\rho$ and states $y_{1}$ and $y_{2}$ we have

$$
\bar{f}_{\theta}(\bar{\theta}(t))=\rho_{1}\left(\tau-\mu C\left(y_{1}\right)\right)^{-1}(-\sin \theta(t)) \rho_{2}\left(\tau-\mu C\left(y_{2}\right)\right)^{-1}(-\sin \theta(t))
$$



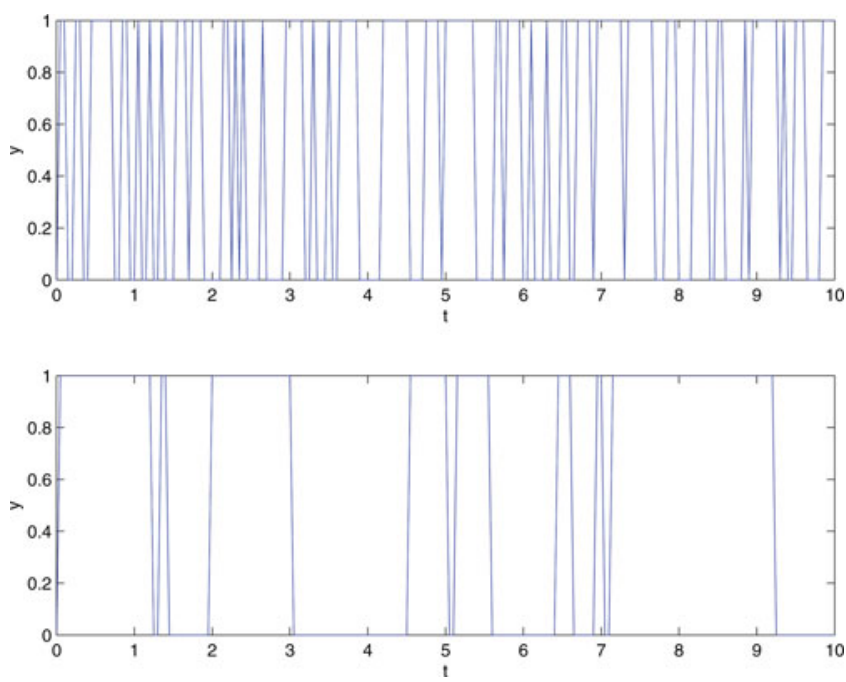

Figure 3. Two-state $\{0,1\}$ Markov path using $\epsilon=0.05$. (top) $a=0.4 \quad b=0.3 \quad$ (bottom) $a=0.04$ $b=0.03$.

Thus using (5.62), (5.63), and (5.64), we have explicit stochastic correction equation given by

$$
\begin{aligned}
d \tilde{\theta}(t)= & \rho_{1}\left(\tau-\mu C\left(y_{1}\right)\right)^{-1}(-\sin \theta(t)) \rho_{2}\left(\tau-\mu C\left(y_{2}\right)\right)^{-1}(-\sin \theta(t)) \tilde{\theta}(t) \\
& +\sigma(t) \sqrt{d t} N(0,1)
\end{aligned}
$$

The stochastic processes

$$
\frac{\theta(t)-\bar{\theta}(t)}{\sqrt{\epsilon}} \approx \tilde{\theta}(t)
$$

converges in expected sense to the solution $\tilde{\theta}(t)$ that is the solution to the integral equation

$$
\widetilde{\theta}(t)=\int_{0}^{t} \bar{f}_{\theta}(s, \omega, \bar{\theta}(s)) \tilde{\theta}(s) d s+\sigma^{2}(t)
$$

which will be studied in future.

A systematic investigation of the effect of noise using Equations (5.54) and (5.58) is given in the next section.

Remark 4. Figure 3 describes the switching behavior from state 0 to 1 and vise versa keeping $\epsilon$ (update) fixed with varying $a$ and $b$.

\section{Discussions}

The SDE with perturbed noise for the $\dot{\theta}$-network model can be used to analyze an aspect of the wave propagation in the network. Thus, we experiment with the $\dot{\theta}$-network model (5.58) using the noise defined by two-state Markov process $y=\{0,1\}$ with transition probability 

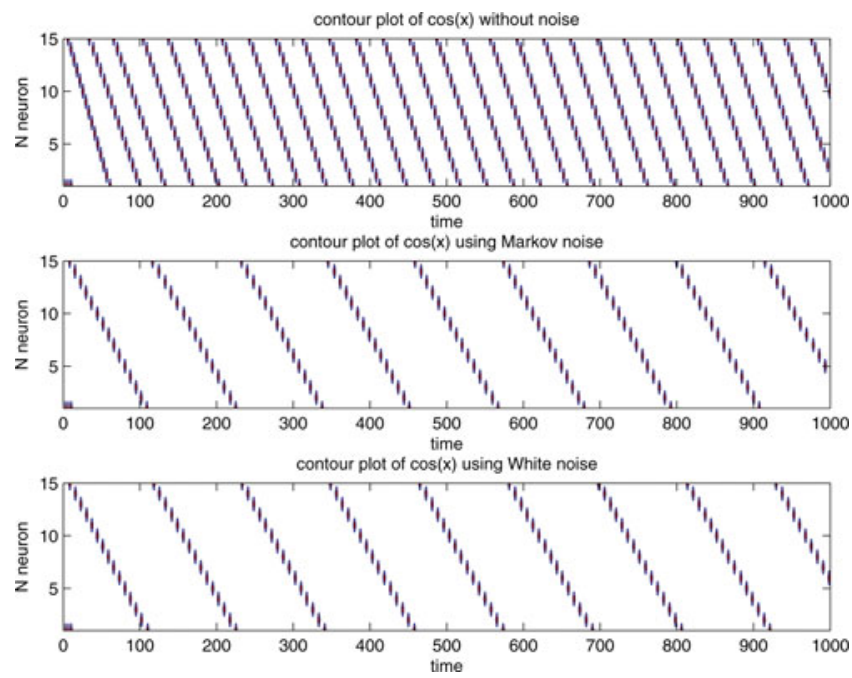

Figure 4. Compare the effect of wave propagation using Markov and Gaussian white noise of $\dot{\theta}$-network model (5.59). Top: A contour plot of $\cos \theta(t)$ without noise is given. Middle: A contour plot using Markov noise generated with $a=0.9, b=0.8$ is given. Bottom: A contour plot of $\cos \theta(t)$ using Gaussian white noise is given.

matrix given by

$$
P=\left[\begin{array}{cc}
1-a & a \\
b & 1-b
\end{array}\right]
$$

for $0<a, b<1$, and its influence constructively in the propagating behavior in the network. We have observed stable waves for any value of $0<a, b<1$ provided $a, b$ is appropriately chosen (not too small).

To see the effect of Gaussian white noise, in Equation (5.58) we choose a non zero mean define by $\bar{y}=\rho_{1} y_{1}+\rho_{2} y_{2}$, and the variance of the system is computed using Equation (5.62). We observe in Equation (5.62) that if $a, b$ are reasonably small, the wave propagation sustains in a detectable way. We have performed numerical simulations of Equation (5.58) for $t \in[0, T]$, where $T$ is the final time within which the wave is confined. Equation (5.58) has been numerically solved using the Forward Euler method with a sufficiently small time step. The noise in the neural network has been assumed to be of the form $C(y(t / \epsilon))$, which corresponds to a circular process. We chose $T=1000$ and $N=15$ so that $\tau$ is a $15 \times 15$ diagonal matrix with all 1's in the upper diagonal, $\omega=[1, \ldots, 1]^{\prime}$ of length $15, \mu=0.1$, and external signal $F(t)=[1,0, \ldots 0]^{\prime}$ of length 15 . We present the result of numerical simulation of (3.21) using the forward Euler method. In Figures 4-6, we show that the noise generated by two-state transition probability matrix described earlier influences constructively in the wavepropagating behavior of the SNLC model (5.58) using Markov and Gaussian white noise. In the context of $\dot{\theta}$-network, numerical results suggest that increasing variances correspond to slow down wave propagation in the network.

The variable $\tilde{\theta}$ of (5.65) is the limit of the quantity $\frac{\theta-\bar{\theta}}{\sqrt{\epsilon}}$ as $\epsilon \rightarrow 0$. The nature of the convergence of this quantity and the sense in which the error in the formula of the expansion of $\theta$ is small are in the sense of distributions over some interval. Future work will address details of weak convergence, strong convergence, and stability of this type of system. Future work will also address the impact of noise on the node's information processing capability determined by signal to noise ratio which can be estimated by spectral methods. 

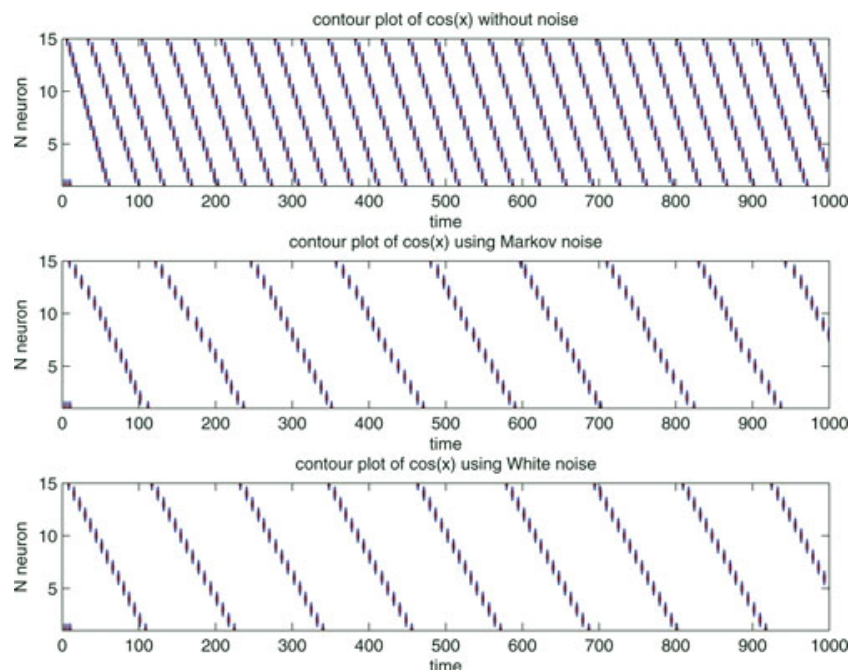

Figure 5. Compare the effect of wave propagation using Markov and Gaussian white noise of $\dot{\theta}$-network model (5.59). Top: A contour plot of $\cos \theta(t)$ without noise is given. Middle: A contour plot using Markov noise generated with $a=0.45, b=0.4$ is given. Bottom: A contour plot of $\cos \theta(t)$ using Gaussian white noise is given.
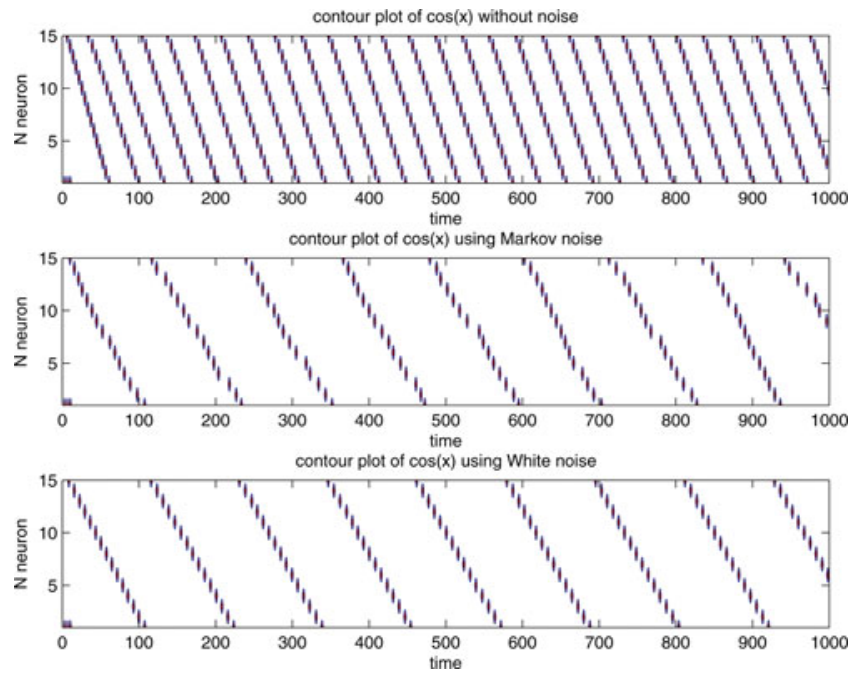

Figure 6. Compare the effect of wave propagation using Markov and Gaussian white noise of $\dot{\theta}$-network model (5.59). Top: A contour plot of $\cos \theta(t)$ without noise. Middle: A contour plot using Markov noise generated with $a=0.225, b=0.2$ is given. Bottom: A contour plot of $\cos \theta(t)$ using Gaussian white noise is given.

\section{Acknowledgments}

The author is grateful to the anonymous referee(s) for their careful reading and useful suggestions that significantly improved the earlier version of the manuscript.

\section{References}

Berman, A., and R. J. Plemmons. 1994. Nonnegative matrices in the mathematical sciences. Classics in Applied Mathematics. Vol. 9. Philadelphia: SIAM. 
Borodin, A. N., and P. Salminen. 2002. Handbook Brownian motion-facts and formulae. 2nd ed. Probability and Its Applications. Boston: Birkhäuser.

Hoppensteadt, F. C., and E. M. Izhikevich. 2006. Synaptic organizations and dynamical properties of weakly connected neural oscillators. Biological Cybernetics 75:117-27.

Hoppensteadt, F. C. 1997. An introduction to the mathematical Neurons. 2nd ed. Cambridge. U.K.: Cambridge University Press.

Karlin, S. 1972. A first course in stochastic processes. USA: Academic press.

Rahman, M., Z. Jackiewicz, and B. D. Welfert. 2007. Stochastic approximation of perturbed Fredholm Volterra integro-differential equation arising in mathematical neurosciences. Applied Mathematics and Computation 186:1173-82.

Rahman, M., and B. Welfert. 2013. Functional central limit theorem for Markov processes and chain. Journal of Probability and Statistical Science (JPSS) 11 (2):111-127.

Romanovsky, V. 1970. Discrete Markov chain. Groningen, Netherlands: Wolters-Noordhoff Publishing.

Skorokhod, A. V., F. C. Hoppensteadt, and H. Salehi. 2002. Random perturbations methods with applications in science and engineering, Applied Mathematical Sciences 150. New York: Springer-Verlag.

Skorokhod, A. V. 2000. On randomly perturbed linear oscillating mechanical systems. Ukrainian Mathematical Journal 52:1483-95. 